\title{
环糊精/氨基粘土超分子水凝胶的构筑及其 $\mathrm{I}_{3}{ }^{-} / \mathrm{I}_{2}$ 吸附性能
}

\author{
牛 杰 ${ }^{a}$ 陈 涌 ${ }^{a}$ 刘 育*,a,b \\ ( ${ }^{a}$ 南开大学化学学院 元素有机化学国家重点实验室 天津 300071) \\ ( ${ }^{b}$ 天津市化学化工协同创新中心 天津 300072)
}

\begin{abstract}
摘要 氨基粘土(Aminoclay, AC)是一种具有片层结构的硅酸盐材料, 由于其在水中具有高分散性的特点，因此被广泛 应用于构筑智能杂化水凝胶. 利用静电相互作用, 将带负电的 7-[6-脱氧-6-(2-乙磺酸)]- $\beta$-环糊精(磺化环糊精, SCD)与氨 基粘土非共价结合，构筑了一种新型的杂化水凝胶，并利用 $\mathrm{X}$ 射线衍射(XRD)、扫描式电子显微镜(SEM)、傅里叶变换 红外光谱(FT-IR)、Zeta 电势、流变测试等对所构筑的凝胶进行了结构表征. 进而发现 SCD-AC 凝胶在水相中展现出对 $\mathrm{I}_{3}{ }^{-}$离子的高效吸附特性, 并且在有机相中能够吸附 $\mathrm{I}_{2}$ 分子.
\end{abstract}

关键词 超分子水凝胶; 环糊精; 氨基粘土; 碘; 吸附

\section{Construction of Cyclodextrin/Aminoclay-Based Supramolecular Hydrogel and Its $\mathrm{I}_{3}{ }^{-} / \mathrm{I}_{2}$ Adsorption Property}

\author{
Niu, Jie ${ }^{a} \quad$ Chen, Yong ${ }^{a} \quad$ Liu, Yu ${ }^{*, a, b}$
}

( ${ }^{a}$ State Key Laboratory of Elemento-Organic Chemistry, College of Chemistry, Nankai University, Tianjin 300071) ( ${ }^{b}$ Collaborative Innovation Center of Chemical Science and Engineering (Tianjin), Tianjin 300072)

\begin{abstract}
Aminoclay (AC), as a kind of layered silicates with positive charges, shows high dispersity in water and has been widely applied in the fabrication of intelligent hybrid hydrogel. Herein, heptakis-[6-deoxy-6-(2-sulfanylethanesulfonic acid)]- $\beta$-cyclodextrin (SCD), a kind of negatively charged cyclodextrin, was employed to construct the supramolecular hydrogel with aminoclay through the static electronic interactions, and the resultant SCD-AC hydrogel was fully characterized by means of X-ray powder diffraction (XRD), scanning electronic microscopy (SEM), fourier transform infrared spectroscopy (FT-IR), zeta potential, rheological test, etc. Significantly, the SCD-AC hydrogel can efficiently absorb not only $\mathrm{I}_{3}{ }^{-}$ion in aqueous solution but also $\mathrm{I}_{2}$ molecule in organic solvent, giving a high adsorption capacity up to $90 \%$ with 10 min.

Keywords supramolecular hydrogel; cyclodextrin; aminoclay; iodine; adsorption
\end{abstract}

水凝胶是一种含水量高、生物相容性好、环境友好 的软材料, 被广泛地应用到组织工程 ${ }^{[1,2]}$ 、药物运输 ${ }^{[3 \sim 6]}$ 、 污染物去除 ${ }^{[7]}$ 以及其它领域 ${ }^{[811]}$ 中. 形成传统水凝胶的 结构单元一般为天然或者合成的高分子化合物, 如聚丙 烯酰胺 ${ }^{[12]}$ 、聚乙烯醇 ${ }^{[13]}$ 、琼脂等天然聚合物 ${ }^{[14 ~ 16]}$, 虽然 这类水凝胶已经被广泛研究并且具有优良的特性，但是 制备过程往往很繁琐, 功能基团的引入也具有一定困 难. 因此, 设计并构筑结构新颖、制备过程简单且具有 优异功能的水凝胶成为了近些年的研究热点.

超分子化学是以分子识别为基础, 利用非共价键
“自下而上”地构筑具有不同功能和性质的分子组装 体系. 这种 “自下而上” 的设计方法在构筑纳米尺度的 分子机器中得到了广泛应用 ${ }^{[17,18]}$, 同时还可以有效地降 低目标体系的合成难度 ${ }^{[19,20]}$, 并且所构筑的超分子体系 往往具备刺激响应的重要性质 ${ }^{[21 ~ 24]}$. 因此, 将超分子化 学方法引入到水凝胶制备中将会为设计合成具有特定 功能的水凝胶体系 ${ }^{[25]}$ 提供了新的思路. 目前, 超分子水 凝胶已经受到了人们的广泛关注, 并展现出在智能窗材 料、自修复材料、光学材料及可控释放等领域的潜在应 用 ${ }^{[26 \sim 31]}$

\footnotetext{
* Corresponding author. E-mail: yuliu@nankai.edu.cn

Received July 29, 2018; revised September 17, 2018; published online September 26, 2018.

Dedicated to Professor Qingyun Chen on the occasion of his 90th birthday.

Project supported by the National Natural Science Foundation of China (Nos. 21432004, 21672113, 21772099, 21861132001, 91527301).

国家自然科学基金(Nos. 21432004, 21672113, 21772099, 21861132001, 91527301)资助项目.
} 
氨基粘土(Aminoclay, AC) 是一种具有三明治结构 的有机粘土, 由 Mann 及其同事 ${ }^{[32]}$ 首次合成并报道. 该 化合物的中心是具有层状硅酸盐结构的镁氧单元, 上下 两边均修饰了氨基. 因此, 在水这种极性大的溶剂中, 氨基会发生质子化, 促使氨基粘土发生层层剥落的现 象, 进而展现出非常高的分散度, 且氨基的存在也使得 其表面带有正电荷. 由于这些独特的性能, 氨基粘土已 经被广泛应用于多个方面, 例如增加金属纳米粒子的稳 定性, 作为支架支撑有机发色团, 制备聚合物一粘土纳 米粒子, 作为模板剂合成柔韧的多孔片层碳材料等. 近 期, 这种新型粘土也被广泛地应用于杂化水凝胶的构筑 中 $^{[33,34]}$.

本文中我们在大环主体分子环糊精的 6 位上引入了 乙基磺酸根, 所形成的 7-[6-脱氧-6-(2-乙磺酸)]- $\beta$-环糊 精(磺化环糊精, SCD)不仅具有明显高于天然环糊精的 水溶性, 而且由于磺酸根的引入也使得磺化环糊精的小 口端具有很高的负电荷密度. 进而, 我们将带有负电荷 的磺化环糊精与表面带正电的氨基粘土进行组装, 构筑 超分子杂化水凝胶. 凝胶自身的网状结构以及环糊精的 空腔使得水凝胶具有吸附污染物的性质. 光谱实验表 明, 我们制备的水凝胶不仅在水溶液中对 $\mathrm{I}_{3}{ }^{-}$离子展现 出了高效率的吸附性能, 其最大吸附量随着磺酸环糊精 的增加而提高, 而且在有机相中也具有一定的吸附 $I_{2}$ 分 子的能力.

\section{2 结果与讨论}

氨基粘土和磺化环糊精根据文献合成 ${ }^{[35,36]}$. 将六水 合氯化镁和 3-氨基丙基三乙氧基硅烷在乙醇中室温摚 拌 $24 \mathrm{~h}$ 后, 收集并洗涤沉淀既可得到氨基粘土, 其结构 由氢核磁共振谱 $\left({ }^{1} \mathrm{H} \mathrm{NMR}\right)$ 和傅里叶变换红外光谱 (FT-IR)验证. X 射线衍射谱图(XRD)在 $2 \theta=5.79^{\circ}$ 处出现
一个强的衍射峰，说明所制备的镁-硅化合物具有片层 结构, 经计算得到的氨基粘土层间距为 $1.6 \mathrm{~nm}$. 透射电 子显微镜(TEM)和扫描电子显微镜(SEM) 图像也均观察 到氨基粘土呈现层状结构 ${ }^{[37]}$.

我们首先研究了水凝胶的形成条件, 在一定体积的 水溶液中, 改变带负电荷的 $\mathrm{SCD}$ 和带正电荷的 $\mathrm{AC}$ 的质 量, 通过倒置小瓶实验来判断凝胶是否形成. 在小瓶中 分别称取氨基粘土 $50 、 60 、 70 、 80 \mathrm{mg}$ 各 6 瓶共 24 瓶，向 每个小瓶中加入 $400 \mu \mathrm{L}$ 去离子水, 加入搅拌子并搅拌 5 $\mathrm{h}$, 直到溶液变得澄清; 同时制备浓度为 $2.66 \mathrm{mg} / \mu \mathrm{L}$ 的 $\mathrm{SCD}$ 水溶液. 以 $50 \mathrm{mg}$ 组为例, 向 6 瓶含有氨基黏土分 散液的小瓶中分别加入 5、10、15、20、25、30 $\mu \mathrm{L} \mathrm{SCD}$ 水溶液后继续搅拌 2 h. $60 、 70 、 80 \mathrm{mg}$ 组均如 $50 \mathrm{mg}$ 组 处理, 同时记录每个小瓶中是否形成凝胶, 汇总整理如 表 1 所述. 由表 1 可以看出, 在 $\mathrm{AC}$ 和 SCD 含量相对较 高的条件下能够形成水凝胶, 并且增加 $\mathrm{AC}$ 的含量能够 降低形成凝胶时需要的 SCD 含量, 这是因为 SCD 小口 端带有较高电荷密度的负电荷, $\mathrm{AC}$ 在水中形成表面带 正电荷的片层剥落物，二者通过静电作用交联而包裹住 水分形成凝胶, 当 $\mathrm{AC}$ 含量较少时, 需要更多的 $\mathrm{SCD}$ 提 供负电荷才能将其交联形成凝胶; 反之, 当 $\mathrm{AC}$ 含量增 加时, 仅需相对较少的 SCD 即能使 AC 交联形成凝胶. 为了保持后续实验的一致性, 我们选择并固定了一个形 成水凝胶的条件 $\left(w_{\mathrm{SCD}}=1.70 \%, w_{\mathrm{AC}}=12.82 \%\right)$ 来进行接 下来的实验.

我们利用流变仪对制得的 SCD-AC 水凝胶的流变 性能进行了测试. 在 $20{ }^{\circ} \mathrm{C}$ 下, 固定应力 $(\gamma)$ 为 $1 \%$ 进行振 荡实验, 可以在图 2 中观察到在一定角频率范围内 $(\omega=$ $0.08 \sim 100 \mathrm{rad} / \mathrm{s})$ 储存模量 $\left(G^{\prime}\right)$ 始终大于损耗模量 $\left(G^{\prime \prime}\right)$, 说明所制备的材料确实具有凝胶的性质. 并且在实验的 角频率范围内储存模量和损耗模量的比值 $(\tan \delta=0.15)$

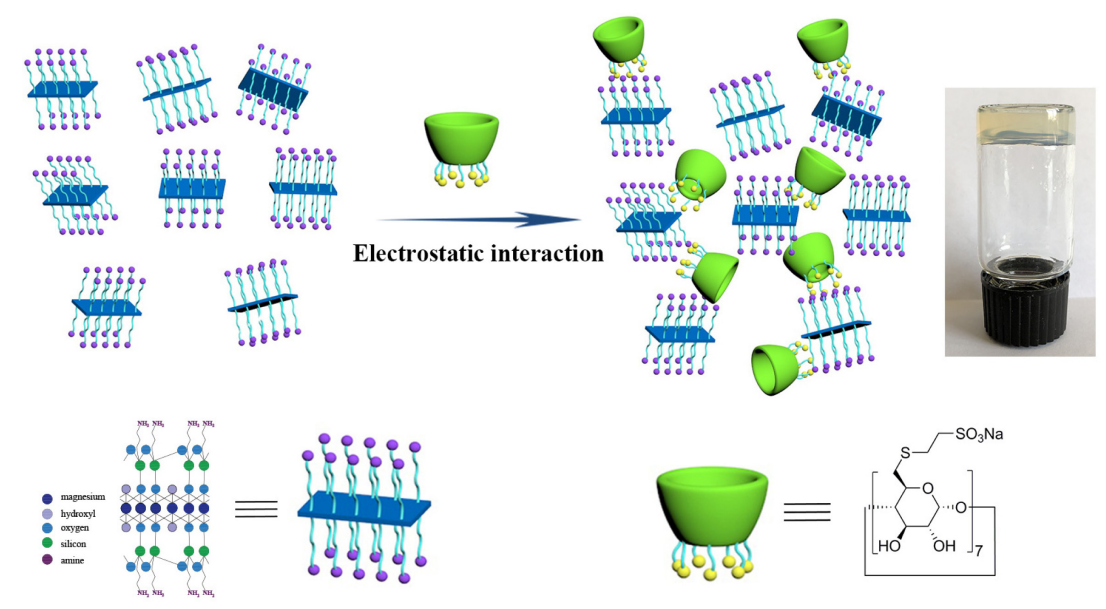

图 1 形成 $\mathrm{SCD}-\mathrm{AC}$ 水凝胶的示意图

Figure 1 Formation of SCD-AC hydrogel 
表 1 成凝条件测试 ${ }^{a}$

Table 1 Test of hydrogel formation

\begin{tabular}{ccccccc}
\hline \multirow{2}{*}{ AC/mg } & \multicolumn{7}{c}{$\mathrm{SCD} / \mathrm{mg}$} \\
\cline { 2 - 7 } & 1.33 & 2.66 & 3.99 & 5.32 & 6.66 & 7.99 \\
\hline 50 & $\mathrm{~S}$ & $\mathrm{~S}$ & $\mathrm{~S}$ & $\mathrm{~S}$ & $\mathrm{~S}$ & $\mathrm{G}$ \\
60 & $\mathrm{~S}$ & $\mathrm{~S}$ & $\mathrm{~S}$ & $\mathrm{~S}$ & $\mathrm{G}$ & $\mathrm{G}$ \\
70 & $\mathrm{~S}$ & $\mathrm{~S}$ & $\mathrm{~S}$ & $\mathrm{G}$ & $\mathrm{G}$ & $\mathrm{G}$ \\
80 & $\mathrm{~S}$ & $\mathrm{~S}$ & $\mathrm{~S}$ & $\mathrm{G}$ & $\mathrm{G}$ & $\mathrm{G}$ \\
\hline
\end{tabular}

${ }^{a}$ For convenience, the SCD and AC contents are calculated in mass (mg), there are 24 samples in total. In the table, the horizontal dimension is the mass of $\mathrm{SCD}$ in each vial, and the longitudinal is the mass of AC in each vial.

没有发生明显变化, 证明 SCD-AC 水凝胶具备一定稳定 性. 另外, 在应变幅度测试中, 固定扫描频率为 $1 \mathrm{~Hz}$, 可以观察到 SCD-AC 水凝胶能够在应变小于 $43 \%$ 时维 持凝胶的性能, 在应变大于 $43 \%$ 之后, 储存模量小于损 耗模量, 凝胶向溶液进行了转变, 也就是说, 凝胶网络 结构发生了破坏. 进而, 我们测试了固定应力为 $2 \%$ 、 $3 \% 、 4 \% 、 5 \% 、 8 \% 、 10 \% 、 15 \%, 20 \% 、 25 \% 、 30 \%$ 下, SCD-AC 水凝胶的振荡频率扫描. 可以观察到在 $8 \%$ 应力范围内, 储存模量、损耗模量及其比值 $\tan \delta$ 均没有明显变化; 应 力由 $10 \%$ 变到 $20 \%$ 的范围内, 储存模量与损耗模量比值

(a)

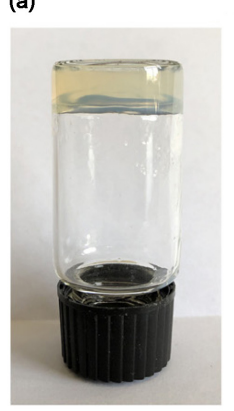

(b)

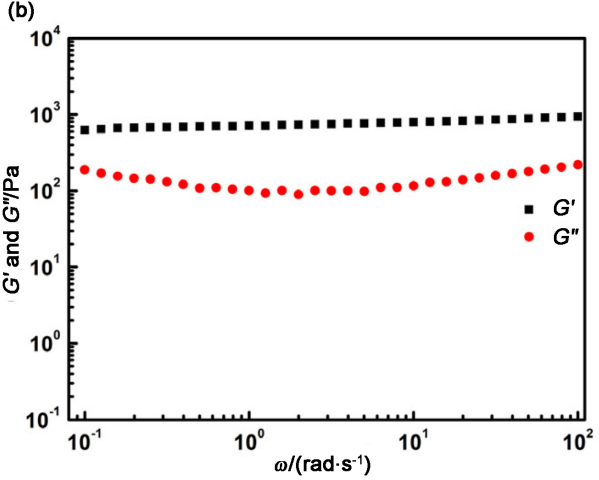

$\tan \delta$ 逐渐减小, 性能逐渐下降; 当应力达到 $25 \%$ 时，储 存模量与损耗模量在高扫描频率下趋近相等; 应力达到 $30 \%$ 时, 储存模量小于损耗模量, 凝胶结构已被破坏. 因此可以推测，在测试振荡频率扫描时，固定应力在 $8 \%$ 范围内, SCD-AC 水凝胶有较好的力学性能, 当固定 应力大于 $25 \%$ 时, 凝胶结构遭到破坏. 此外, 我们还测 试了固定扫描频率为 $2 、 5 、 10 、 20 、 30 、 40 、 50 \mathrm{~Hz}$ 下, $\mathrm{SCD}-\mathrm{AC}$ 水凝胶的应变幅度扫描. 可以观察到在固定扫 描频率 $30 \mathrm{~Hz}$ 以内时，水凝胶的应力扫描图在应力达到 约 $40 \%$ 时, 可以维持凝胶的性能; 在固定频率为 $40 \mathrm{~Hz}$ 时, 这一转变应力变为 $30 \%$; 在固定频率 $50 \mathrm{~Hz}$ 时, 应 变幅度图已无法进行分析. 因此 SCD-AC 水凝胶可以在 固定频率 $40 \mathrm{~Hz}$ 以内进行应变幅度扫描，并且可以在应 力达到 $30 \%$ 左右时保持凝胶结构.

随后，我们将水凝胶冻干后的样品进行 XRD 测试. 如图 3 所示, 与单纯的 $\mathrm{AC}$ 的 XRD 谱图相比, SCD-AC 水凝胶的小角衍射峰有显著增强，同时其它的特征衍射 峰 ${ }^{[33]}$ 没有明显变化, 这证明了在形成凝胶的过程中，氨 基粘土的片层结构基本没有改变. 通过计算，我们发现

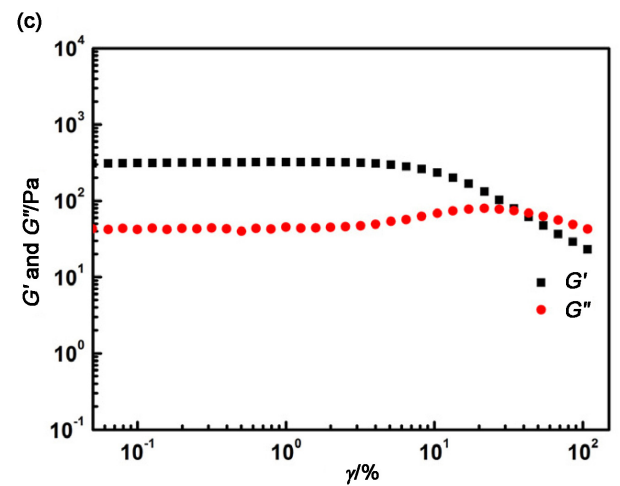

图 2 (a) SCD-AC 水凝胶的照片、(b)固定应力为 $1 \%$ 时 SCD-AC 水凝胶振荡频率扫描图、(c)固定扫描频率为 $1 \mathrm{~Hz}$ 时 SCD-AC 水 凝胶应力扫描图

Figure 2 (a) Image of SCD-AC hydrogel, (b) oscillation frequency sweep of SCD-AC hydrogel at a constant strain of 1\%, and (c) strain sweep of SCD-AC hydrogel at a constant frequency of $1 \mathrm{~Hz}$

Storage modulus $\mathrm{G}^{\prime}(\boldsymbol{\bullet})$, loss modulus $\mathrm{G}^{\prime \prime}(\bullet)$. All the experiments were carried out at $20{ }^{\circ} \mathrm{C}$

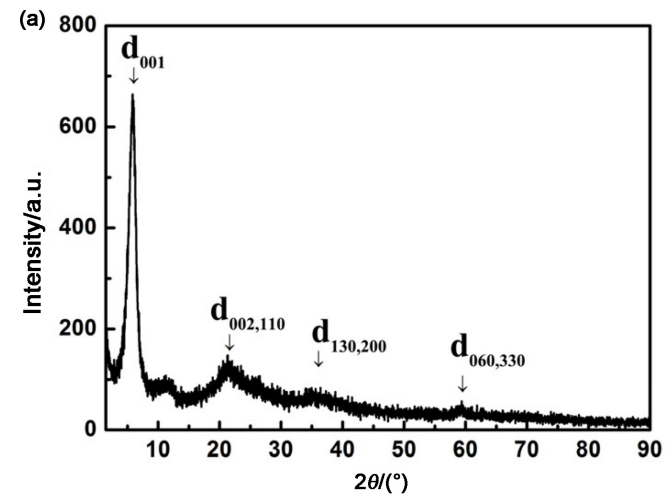

(b)

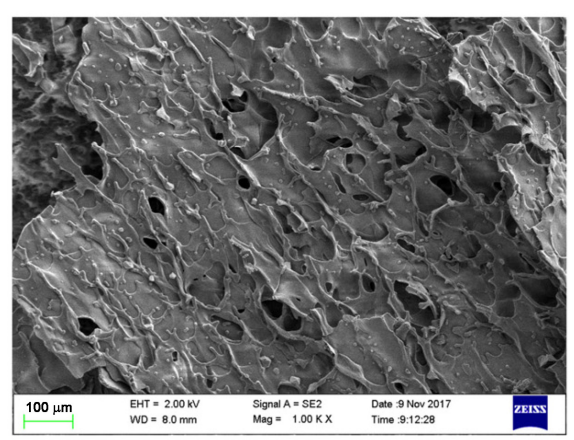

图 $3 \mathrm{SCD}-\mathrm{AC}$ 水凝胶的(a) XRD 谱图和(b) SEM 图像

Figure 3 (a) XRD pattern and (b) SEM image of the SCD-AC hydrogel 
形成水凝胶之后，层间距由以前的 $1.6 \mathrm{~nm}$ 增加到 1.8 $\mathrm{nm}$, 可能是由于带相反电荷的环糊精进入到氨基粘土 的层与层之间, 增加了粘土层间的距离. 从 SEM 图像 中, 我们可以观察到干凝胶呈现出有孔的片状结构, 表 明在形成凝胶的过程中 $\mathrm{AC}$ 的片层结构得以保持. 对比 $\mathrm{AC}$ 和 SCD-AC 水凝胶的红外谱图, 可以观察到 $\mathrm{Mg}-\mathrm{O}$ 伸缩振动(535.05 $\left.\mathrm{cm}^{-1}\right), \mathrm{Si}-\mathrm{O}-\mathrm{Si}$ 振动(1119.53 $\left.\mathrm{cm}^{-1}\right)$ 等特征吸收峰没有明显变化, 进一步说明氨基粘土的结 构在水凝胶中得以保持. 我们推断 SCD 和 $\mathrm{AC}$ 之间的静 电作用力是凝胶形成的主要驱动力, 通过静电作用诱导 $\mathrm{AC}$ 之间发生层层交联, 最后形成了具有多孔结构的杂 化水凝胶. 最后, 我们还对制备的凝胶的表面电荷分布 进行了研究, 测得其 zeta 电势为 $+11.58 \mathrm{mV}$. 结合以上 的实验, 我们可以归纳出制备的 SCD-AC 水凝胶具有以 下特性: (1)具有多孔的结构; (2)表面呈现正电性; (3)环 糊精的空腔没有被占用. 这使得我们制备的水凝胶在吸 附污染物方面有着潜在的应用价值, 尤其对一些疏水或 带负电的化合物.

碘是人身体中一种重要的微量元素, 超过一定含量 会对人体造成危害和腐蚀, 并且过量的碘也会破坏环 境, 造成辐射破坏生态平衡. 但是目前报道的吸附碘的 材料, 如多孔材料 MOFs 只能在有机相中吸附碘, 在水 相中这些材料的性能大大降低, 近期有文献报道利用超
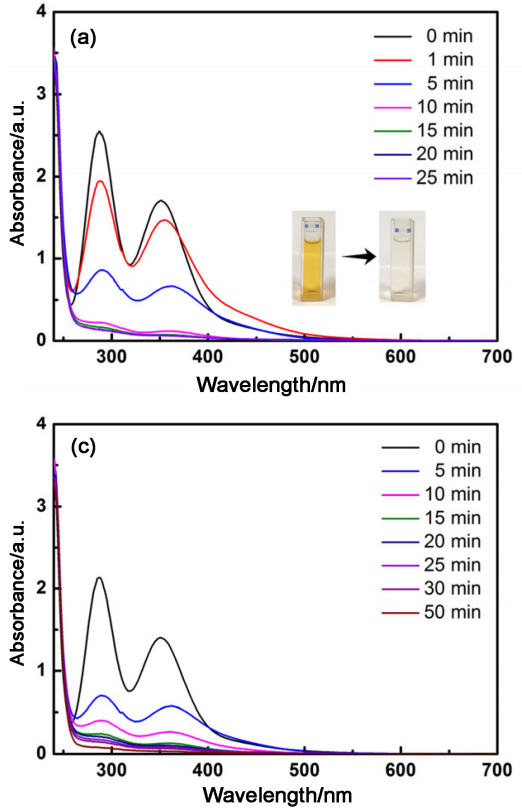

分子体系吸附碘，但是在水相中吸附和去除碘元素仍然 是一个难题 $\left.{ }^{[38} 40\right]$. 众所周知, $I_{2}$ 单质在水中的溶解度很 低，在水相中会结合 $\mathrm{I}^{-}$形成 $\mathrm{I}_{3}{ }^{-}$离子来增大溶解度，对 $\mathrm{I}_{3}{ }^{-}$离子的吸附研究对去除水溶液中富余的 $\mathrm{I}_{2}$ 具有一定 的指导意义. 令我们惊奇的是, SCD-AC 水凝胶在水溶 液中对 $\mathrm{I}_{3}{ }^{-}$离子具有很好的吸附效果.

$\mathrm{KI}-\mathrm{I}_{2}\left([\mathrm{KI}] /\left[\mathrm{I}_{2}\right]=6 / 1\right)$ 溶液的紫外-可见吸收光谱在 288 和 $351 \mathrm{~nm}$ 处出现两个主要的特征吸收峰. 为了消除 在后续实验中散射信号的干扰，我们选取了 $288 \mathrm{~nm}$ 处 的吸收峰强度得到 $\mathrm{I}_{3}{ }^{-}$离子在水溶液中的浓度标准曲线. 随后在水溶液研究了 SCD-AC 水凝胶对 $\mathrm{I}_{3}{ }^{-}$离子的吸附 量以及吸附效率. 将 $312 \mathrm{mg}$ 水凝胶 $\left(w_{\mathrm{SCD}}=1.70 \%, w_{\mathrm{AC}}\right.$ $=12.82 \%$ )加入到 $\mathrm{I}_{3}{ }^{-}$离子水溶液 $(0.029 \mathrm{mg} / \mathrm{mL}, 100 \mathrm{~mL})$ 中, 水溶液的颜色很快从棕红色变为无色，同时凝胶的 颜色由白色变为黄色. 随后利用紫外-可见光谱实验对 水凝胶吸附效率进行了研究，在相同条件下，通过 288 $\mathrm{nm}$ 处的吸收峰强度随时间的变化情况来监测凝胶的吸 附过程. 结果表明, SCD-AC 凝胶可以从水溶液中快速 地去除碘. 从图 4 可以看出, SCD-AC 凝胶在 $10 \mathrm{~min}$ 内 对水溶液中 $\mathrm{I}_{3}{ }^{-}$的吸附效率可以达到 $90 \%$, 并且在 20 $\min$ 内达到接近 100\%的吸附. 当减少用量至 $101 \mathrm{mg}$ 水 凝胶，凝胶的吸附速率有所降低，吸附效率在 $15 \mathrm{~min}$ 内 达到 $90 \%$, 在 $30 \mathrm{~min}$ 内达到接近 100\%(图 4). 上述实验
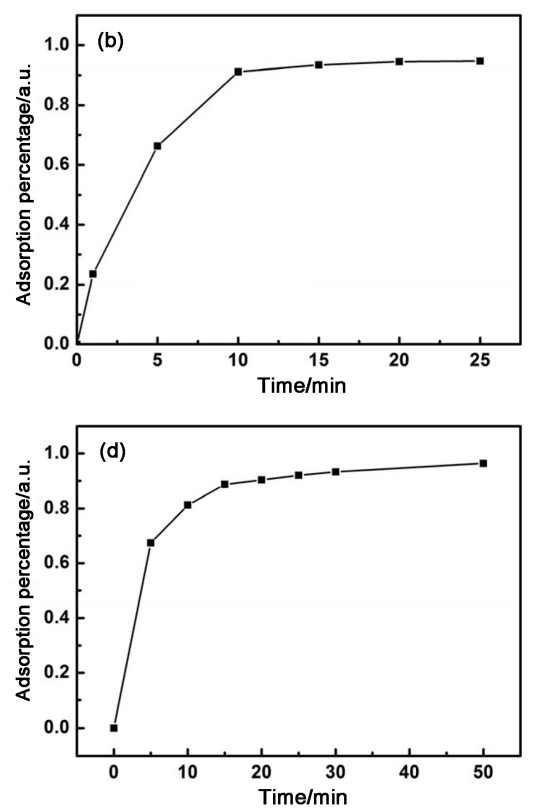

图 4 (a)加入 $312 \mathrm{mg}$ 水凝胶后 $\mathrm{I}_{3}{ }^{-}$溶液的随时间变化的紫外-可见吸收谱图(内置: 吸附前后碘溶液的图像)、(b)在 $288 \mathrm{~nm}$ 处随时 间变化的吸附效率、(c)加入 $101 \mathrm{mg}$ 水凝胶后 $\mathrm{I}_{3}{ }^{-}$溶液的随时间变化的紫外-可见吸收谱图和(d)在 $288 \mathrm{~nm}$ 处随时间变化的吸附效 率

Figure 4 (a) Time-dependent UV/vis absorption spectra of $\mathrm{I}_{3}{ }^{-}$aqueous solution upon addition of $312 \mathrm{mg}$ SCD-AC hydrogel (inset: images of $\mathrm{I}_{3}^{-}$aqueous solution before and after adsorption is taken), (b) time-dependent $\mathrm{I}_{3}{ }^{-}$adsorption percentage at $288 \mathrm{~nm}$, (c) time-dependent $\mathrm{UV} / \mathrm{vis}$ absorption spectra of $\mathrm{I}_{3}{ }^{-}$aqueous solution upon addition of $101 \mathrm{mg} \mathrm{SCD}-\mathrm{AC}$ hydrogel, and (d) time-dependent $\mathrm{I}_{3}{ }^{-}$adsorption percentage at $288 \mathrm{~nm}$ 
表明 SCD-AC 超分子水凝胶在水溶液中展现出非常高 的对 $\mathrm{I}_{3}{ }^{-}$的吸附效率.

我们研究了 SCD-AC 水凝胶在水溶液对 $\mathrm{I}_{3}{ }^{-}$的最大 吸附量. 与上述实验相似, 将 $155 \mathrm{mg}$ 水凝胶 $\left(w_{\mathrm{SCD}}=\right.$ $\left.1.70 \%, w_{\mathrm{AC}}=12.82 \%\right)$ 加入到 $\mathrm{I}_{3}{ }^{-}$离子水溶液 $(0.76 \mathrm{mg} /$ $\mathrm{mL}, 40 \mathrm{~mL}$ )中, 避光摚拌 $24 \mathrm{~h}$. 由于 $\mathrm{I}_{3}{ }^{-}$离子母液浓度过 高, 将母液稀释 15 倍后用于测试紫外-可见吸收光谱测 试(图 S14), 得到 SCD-AC 水凝胶对 $\mathrm{I}_{3}{ }^{-}$最大吸附量为每 $1 \mathrm{mg} \mathrm{SCD}-\mathrm{AC}$ 水凝胶吸附 $0.095 \mathrm{mg}$ 碘 $\mathrm{I}_{3}{ }^{-}$.

有意义的是, 增加凝胶中磺化环糊精的相对含量, 可以线性地提高 $\mathrm{I}_{3}{ }^{-}$的最大吸附量. 制备编号为 $1 、 2 、 3 、$ $4 、 5$ 的五组凝胶, 分别称取 $20 \mathrm{mg} \mathrm{AC}$ 于每组小瓶中, 并 加入 $133 \mu \mathrm{L}$ 去离子水, 搅拌后, 分别向 $1 、 2 、 3 、 4 、 5$ 号小瓶中加入 $1.33,2.66,5.32,7.99,10.65 \mathrm{mg} \mathrm{SCD}$, 制 备出一系列仅环糊精含量不同的杂化水凝胶. 将凝胶加 到 $\mathrm{I}_{3}{ }^{-}$离子水溶液中, 避光搅拌 $12 \mathrm{~h}$, 利用上述方法, 测 量吸附前后溶液的紫外-可见吸收光谱, 计算得每组凝 胶对 $\mathrm{I}_{3}{ }^{-}$的最大吸附量. 并将每组凝胶中 $\mathrm{AC}$ 质量、 $\mathrm{SCD}$ 质量、对 $\mathrm{I}_{3}{ }^{-}$的最大吸附质量整理于表 2 中, 发现随着磺 化环糊精的含量不断增加, $\mathrm{I}_{3}{ }^{-}$的最大吸附量也随之增 加, 并呈现出线性的关系. 这个结果表明我们制备的凝 胶对 $\mathrm{I}_{3}{ }^{-}$的吸附不仅仅是靠氨基粘土和 $\mathrm{I}_{3}{ }^{-}$离子之间的静 电作用, 环糊精在吸附过程中也起着至关重要的作用.

表 2 凝胶中磺化环糊精含量对最大吸附量的影响 Table 2 SCD content of hydrogels and the max adsorption quantity

\begin{tabular}{cccccc}
\hline Quantity/mg & 1 & 2 & 3 & 4 & 5 \\
\hline AC & 20 & 20 & 20 & 20 & 20 \\
SCD & 1.33 & 2.66 & 5.32 & 7.99 & 10.65 \\
Max adsorption & 14.75 & 15.79 & 16.46 & 17.64 & 19.08 \\
\hline
\end{tabular}

除在水溶液中吸附 $\mathrm{I}_{3}{ }^{-}$之外, $\mathrm{SCD}-\mathrm{AC}$ 凝胶还能够在 有机溶剂中吸附 $\mathrm{I}_{2}$ 分子. 由于水和正己烷不相溶, 我们 使用了 SCD-AC 干凝胶进行了以下实验. 先利用紫外可见吸收光谱 $523 \mathrm{~nm}$ 处的吸收峰, 计算得到了 $\mathrm{I}_{2}$ 在正 己烷中的标准曲线. 然后将 $10 \mathrm{mg}$ 干凝胶加到碘 $\left(\mathrm{I}_{2}\right)$ 的 正己烷溶液 $(0.094 \mathrm{mg} / \mathrm{mL}, 3 \mathrm{~mL})$ 中, 测试一定时间间隔 的正已烷溶液的紫外-可见吸收光谱, 利用 $523 \mathrm{~nm}$ 处的 吸收峰计算碘 $\left(\mathrm{I}_{2}\right)$ 的浓度, 进而计算凝胶对碘 $\left(\mathrm{I}_{2}\right)$ 的吸附 速率. SCD-AC 凝胶在 $390 \mathrm{~min}$ 内能够吸附正己烷溶液 中约 $60 \%$ 的 $\mathrm{I}_{2}$, 其效率略低于 SCD-AC 凝胶在水溶液中 对 $\mathrm{I}_{3}{ }^{-}$的吸附效率. 将 $9.39 \mathrm{mg}$ 干凝胶加到碘 $\left(\mathrm{I}_{2}\right)$ 的正己 烷溶液 $(0.094 \mathrm{mg} / \mathrm{mL}, 3 \mathrm{~mL})$ 中, 避光放置 $12 \mathrm{~h}$, 计算得 到干凝胶对碘 $\left(\mathrm{I}_{2}\right)$ 的最大吸附量为每 $1 \mathrm{mg}$ 干凝胶吸附 $0.19 \mathrm{mg} \mathrm{I}$. 上述实验表明 SCD-AC 凝胶可以在有机溶 剂中吸附碘 $\left(\mathrm{I}_{2}\right)$, 进一步扩展了这类基于大环主体的超 分子凝胶的应用范围.

\section{2 结论}

在本文中, 我们利用一种带负电的环糊精和带正电 的氨基粘土成功地构筑了杂化超分子凝胶，该凝胶展现 出了一定的力学性质. 由于凝胶表面正电性和环糊精空 腔的引入，使得这类凝胶在污染物的吸附方面有着潜在 的应用. 随后的吸附碘的实验证明, SCD-AC 凝胶不但 能在水溶液中实现对 $\mathrm{I}_{3}{ }^{-}$的快速高效去除, 而且在有机 溶剂中展现出一定的吸附 $\mathrm{I}_{2}$ 的能力. 本文的研究可以为 今后构筑具有功能基团的杂化凝胶提供新的思路.

\section{3 实验部分}

\section{1 仪器与试剂}

核磁共振波谱仪为 Bruker AV400 spectrometer; 紫 外-可见分光光谱仪为 Shimadzu UV-3600 spectrometer (温控装置: PTC-348WI), 采用光路长度为 $1 \mathrm{~cm}$ 的石英 比色血; 扫描电镜(SEM): 采用 JSM-7500F 扫描电子显 微镜; 利用移液枪吸取 $5 \mu \mathrm{L}$ 样品滴于盖玻片上，自然晾 干; 透射电镜(TEM): FEI Tecnai G2 F20, 加速电压 200 $\mathrm{kV}$. 束斑漂移率 $0.675 \mathrm{~nm} / \mathrm{min}$; 图像分辨率: 点分辨率 $0.24 \mathrm{~nm}$, 线分辨率 $0.102 \mathrm{~nm}$. 利用移液枪吸取 $5 \mu \mathrm{L}$ 样 品滴于碳支撑膜铜网上, 自然晾干. 流变仪: AR 2000ex (TA Instrument)系统上使用 $40 \mathrm{~mm}$ 平板进行流变学测试; Zeta 电位: 在 Zeta PALS + BI-90 instrument (Brookhaven Company)电位仪、 $25{ }^{\circ} \mathrm{C}$ 条件下测定; Rigaku D/max$2500 \mathrm{X}$ 射线衍射仪在 $18 \mathrm{~kW}, 60 \mathrm{kV}, 300 \mathrm{~mA}$ 下测试; 红 外光谱(FT-IR)为 Bruker TENSOR II.

\section{2 实验方法}

\subsubsection{SCD-AC 水凝胶的合成}

称取 $60 \mathrm{mg}$ 氨基粘土于小瓶中, 加入 $400 \mu \mathrm{L}$ 去离子 水, 并加入搅拌子搅拌 $5 \mathrm{~h}$, 直到溶液变得澄清, 向小瓶 中加入 $30 \mu \mathrm{L}$ 浓度为 $0.266 \mathrm{mg} / \mu \mathrm{L}$ 的磺化环糊精水溶液, 后继续摚拌 $2 \mathrm{~h}$, 其间氨基黏土逐渐发生交联, 溶液逐 渐向凝胶状态转变, 静置 $2 \mathrm{~h}$ 使凝胶充分交联, 即形成 了水凝胶.

辅助材料(Supporting Information) 提供了磺化环糊 精和氨基粘土的基本表征以及水凝胶的吸附实验数据. 这些材料可以免费从本刊网站(http://sioc-journal.cn/)上 下载.

\section{References}

[1] Lee, K. Y.; Mooney, D. J. Chem. Rev. 2001, 101, 1869.

[2] Park, M.-R.; Seo, B.-B.; Song, S.-C. Biomaterials 2013, 34, 1327.

[3] Peppas, N. A.; Hilt, J. Z.; Khademhosseini, A.; Langer, R. $A d v$. Mater. 2006, 18, 1345.

[4] Jianyu, L.; Eckhard, W.; Sabine, G.-G.; Michael, S.; Andreas, K.; 
Christine, H.; Nathalie, A.; Arno, D.; Anne, B.; Brno, T.; Kuno, W.; Mara, F.; Michaela, K.; Alexander, S.; R., F. B.; J., M. D. $A d v$. Healthcare Mater 2018, 7, 1701393.

[5] Qiu, Y.; Park, K. Adv. Drug Delivery Rev. 2001, 53, 321.

[6] Ma, X.-Y.; Shi, L. J.; Zhou, J.; Zhu, J.; Zhong, J.; Wei, R. L. Chin. J. Org. Chem. 2013, 33, 1080 (in Chinese). (马晓晔, 施丽君, 周滑, 朱君, 钟建, 魏锐利, 有机化学, 2013, 33, 1080.)

[7] Gan, L.; Li, H.; Chen, L.; Xu, L.; Liu, J.; Geng, A.; Mei, C.; Shang, S. Colloid Polym. Sci. 2018, 296, 607.

[8] Yuri, N.; Itaru, S.; Yoshimi, S.; Yoshio, N. Chem. Lett. 2007, 36, 1204.

[9] Guo, Y.; Wang, R.; Shang, Y.; Liu, H. RSC Adv. 2018, 8, 8699.

[10] Shih, I.-L.; Shen, M.-H.; Van, Y.-T. Bioresour. Technol. 2006, 97, 1148 .

[11] Niu, Y.; Guo, T.; Yuan, X.; Zhao, Y.; Ren, L. Soft Matter 2018, 14, 1227.

[12] Lee, K.; Asher, S. A. J. Am. Chem. Soc. 2000, $122,9534$.

[13] Kobayashi, M.; Toguchida, J.; Oka, M. J. Biomed. Mater. Res. 2001, 58, 344

[14] Hamidi, M.; Azadi, A.; Rafiei, P. Adv. Drug Delivery Rev. 2008, 60, 1638

[15] Lehr, C.-M.; Bouwstra, J. A.; Schacht, E. H.; Junginger, H. E. Int. J. Pharm. 1992, 78, 43.

[16] Jeong, B.; Kim, S. W.; Bae, Y. H. Adv. Drug Delivery Rev. 2002, 54, 37.

[17] Shi, H.; Cheng, X. B.; Lin, Q.; Yao, H.; Zhang, Y. M.; Wei, T. B. Chin. J. Org. Chem. 2018, 38, 1718 (in Chinese). (师海雄, 程晓斌, 林奇, 姚虹, 张有明, 魏太保, 有机化学, 2018, 38, 1718.)

[18] Han, Y.-Q.; Zhou, W. J.; Shen, H. M.; Liu, Q. P.; Yu, W. Y.; Ji, H. B.; She, Y. B. Chin. J. Org. Chem. 2016, 36, 248 (in Chinese). (韩叶强, 周文杰, 沈海民, 刘秋平, 于文艳, 纪红兵, 余远斌, 有机化学, 2016, 36, 248.)

[19] Kay, E. R.; Leigh, D. A.; Zerbetto, F. Angew. Chem., Int. Ed. 2007, 46, 72 .

[20] Daniel, M.-C.; Astruc, D. Chem. Rev. 2004, 104, 293.

[21] Yu, J.; Zhang, Y.-M.; Li, P.-Y.; Liu, Y. Chem. Commun. 2017, 53, 3717.
[22] Zeng, F.; Zimmerman, S. C. Chem. Rev. 1997, 97, 1681.

[23] Sangeetha, N. M.; Maitra, U. Chem. Soc. Rev. 2005, 34, 821.

[24] Wang, C.-H.; Tang, Q.; Xi, Y.-Y.; Yang, M.; Li, T.; Huang, Y.; Tao, Z. Chin. J. Org. Chem. 2018, 38, 1394 (in Chinese). (王成会，唐青，席芸芸，杨梅，李涛，黄英，陶朱，有机化学， 2018, 38, 1394.)

[25] Zhao, Q.; Li, S. H.; Liu, Y. Prog. Chem. 2018, 30, 673 (in Chinese). (赵倩, 李盛华, 刘育, 化学进展, 2018, 30, 673.)

[26] Wang, X.; Wang, J.; Yang, Y.; Yang, F.; Wu, D. Polym. Chem. 2017, 8,3901 .

[27] Zhao, Q.; Chen, Y.; Li, S.-H.; Liu, Y. Chem. Commun. 2018, 54, 200 .

[28] Li, Z.; Zhang, Y.-M.; Wang, H.-Y.; Li, H.; Liu, Y. Macromolecules 2017, 50, 1141.

[29] Liu, G.; Zhang, Y. M.; Xu, X.; Zhang, L.; Liu, Y. Adv. Opt. Mater. 2017, 5, 1700149 .

[30] Chen, J.-F.; Liu, X.; Han, B.-B.; Ding, J.-D.; Zhang, Y.-M.; Lin, Q.; Yao, H.; Wei, T.-B. Chin. J. Org. Chem. 2018, 38, 2741 (in Chinese).

(陈进发，刘茜，韩冰冰，丁金东，张有明，林奇，姚虹，魏太保， 有机化学, 2018, 38, 2741.)

[31] Zhao, Q.; Chen, Y.; Liu, Y. Chin. Chem. Lett. 2018, 29, 84.

[32] Burkett, S. L.; Press, A.; Mann, S. Chem. Mater. 1997, 9, 1071.

[33] Rao, K. V.; Datta, K. K. R.; Eswaramoorthy, M.; George, S. J. Adv. Mater. 2013, 15, 1713.

[34] Wang, T.; Li, P.; Li, H. ACS Appl. Mater. Interfaces 2014, 6, 12915.

[35] Steffen, A.; Thiele, C.; Tietze, S.; Strassnig, C.; Kämper, A.; Lengauer, T.; Wenz, G.; Apostolakis, J. Chem.-Eur. J. 2007, 13 6801.

[36] Wenz, G.; Strassnig, C.; Thiele, C.; Engelke, A.; Morgenstern, B.; Hegetschweiler, K. Chem.-Eur. J. 2008, 14, 7202.

[37] Datta, K. K. R.; Achari, A.; Eswaramoorthy, M. J. Mater. Chem. A 2013, 1,6707 .

[38] Zeng, M.-H.; Wang, Q.-X.; Tan, Y.-X.; Hu, S.; Zhao, H.-X.; Long, L.-S.; Kurmoo, M. J. Am. Chem. Soc. 2010, 132, 2561.

[39] Liu, Q.-K.; Ma, J.-P.; Dong, Y.-B. Chem. Commun. 2011, 47, 7185.

[40] Jie, K.; Zhou, Y.; Li, E.; Li, Z.; Zhao, R.; Huang, F. J. Am. Chem. Soc. 2017, 139, 15320 .

(Cheng, F.) 